\title{
SOME NONLINEAR FUNCTIONALS FOR SCALAR CONSERVATION LAWS
}

\author{
TAI-PING LIU*, TONG YANG ${ }^{\dagger}$, AND YONGSHU ZHENG ${ }^{\ddagger}$
}

\begin{abstract}
In this paper, we will first summarize our recent study on nonlinear functionals which capture the nonlinear behaviour and entropy production of solutions for scalar conservation laws. These functionals are instrumental in the sudy of the behavior of the characteristic fields in systems of conservation laws. The application of these functionals to the problem of the uniform boundedness and stability in $L_{1}$-norm of the weak solutions to systems of hyperbolic conservation laws is briefly presented. Then we will generalize one of the functionals to viscous scalar conservation laws and study the relation between the invisid and viscous cases for nonlinear waves.
\end{abstract}

1. Introduction. In this paper, we will study some nonlinear functionals for scalar conservation laws and discuss their relation with some nonlinear properties of the weak solutions to systems. The scalar conservation law has been well investigated and there is a satisfactory well-posedness theory. Nevertheless, the introduction of some of these functionals is new and their application to the study of systems of hyperbolic conservation has been essential, [11, 13, 14].

Consider the initial value problem for the scalar hyperbolic conservation law

$$
\begin{aligned}
& u_{t}+f(u)_{x}=0 \\
& u(x, 0)=u_{0}(x)
\end{aligned}
$$

Without loss of generality, we assume $f^{\prime}(0)=0$. It is well-known that, even for smooth initial data, solution usually can not be smooth and we need to consider weak solution satisfying

$$
\iint\left(u(x, t) \phi_{t}(x, t)+f(u(x, t)) \phi_{x}(x, t)\right) d x d t+\int u_{0}(x) \phi(x, 0) d x=0,
$$

for every test function $\phi(x, t) \in C_{0}^{1}\left(R \times R^{+}\right)$. It is also well-known that solutions for the initial value problem of (1.1) satisfying (1.2) is not unique and certain entropy condition is needed to choose a unique physical solution. For example, when the flux function is convex, a sufficient condition requires that

$$
\eta(u)_{t}+q(u)_{x} \leq 0
$$

in the weak sense. Here the entropy pair $(\eta(u), q(u))$ satisfies $q^{\prime}(u)=\eta^{\prime}(u) f^{\prime}(u)$ so that the inequality becomes an equality when the solution is smooth. For the complete study of the well-posedness theory for scalar conservation laws see [6]. It is known that, for convex flux, the time derivative of the integral of the entropy $\eta(u)$ with respect to $x$ on $R$ is equivalent to the negation of the third power of the strength of the shock waves in the solutions at the time, $[7,8]$. This property is crucially used in the study of the compactness property of the solution operator by the theory of compensated compactness, [20]. Notice that the entropy inequality is effective only when there are shock waves in the solution. Since $u^{2}$ is one of the convex entropies of (1.1), the $L_{2}$-norm of a solution has the property mentioned above. Further study

\footnotetext{
*Department of Mathematics, Stanford University.

†Department of Mathematics, City University of Hong Kong.

${ }^{\ddagger}$ Department of Mathematics, Hua Qiao University.
} 
on the $L_{2}$-norm of a solution when the flux function is nonconvex shows the similar property, cf. [14]. In fact, in terms of the bifurcation of the Hugoniot curve from the rarefaction curve of the same family in a system, the properties for convex and noncovex have the same expressions.

A major shortcoming of this basic functional is that it can not be generalized to the study of two solutions because the $L_{2}$-norm of the difference of two solutions of (1.1) may increase at an arbitrary rate. This is because the $L_{2}$-norm decreases due to dissipation and there are anti-dissipations when compression waves are present in the solutions. On the other hand, it is known that the solution operator is a $L_{1}$ contraction semigroup. However, the $L_{1}$ topology is insensitive to the nonlinearity of the partial differential equation and stays constant unless one of the solutions crosses the other at a shock, [15].

An effective nonlinear functional is defined in [12] which is in terms of the $L_{1}$ difference of two solutions and the variation of each of the solutions when the flux function is convex. It decreases in time even when the solutions are smooth and reveals the nonlinear evolution of the coupling of the wave patterns of the two solutions. In fact, it captures the basic nonlinearity of the flux function $f(u)$. In this paper, we study the effect of viscosity to this functional. We consider the generalization of the functional to the viscous scalar conservation law and obtain its time derivative. Since this functional captures the essential nonlinearity of the conseravtion law, it decreases more rapidly for the compression waves than for the shock of the same strength. The understanding of this in the viscous case allows us to study the relation between the decreasing rates for the viscous case and for the invisid case in a simple situation with shocks. Rigorous analysis on this relation for general solutions is beyond the scope of this paper and will be pursued by the authors in the future.

The above nonlinear functionals on weak solutions to scalar conservation laws are closely related to the study of weak solutions to systems. When we consider the coupling of waves to a system of hyperbolic conservation laws, it is natural to study the bifurcation of the Hugoniot curve from the rarefaction curve of the same family. In fact, if the Hugoniot curve coincides with the rarefaction curve, cf. [19], the system behaves like a set of scalar conservation laws. This bifurcation is controlled by the derivative of the square of the $L_{2}$-norm of the solution. Thus, the $L_{2}$-norm of the weak solution to a system, when combined with other nonlinear functionals on the coupling of waves in different families and wave interaction potential, can be used to yield the uniform boundedness of weak solutions in $L_{1}$-norm, cf. [11, 14]. When we consider the relation between two weak solutions to a system, it is crucial to estimate the distance between the end states of a wave in one solution and the state given by the other solution. If the distance is measured by the Hugoniot curves of the same family, then it is shown in [12] that the time rate of increase of $L_{1}$ distance can be controlled by the time derivative of this generalized entropy functional and one of its applications is given to the study of $L_{1}$ stability of weak solutions to systems in [13].

The rest of the paper is organized as follows. In Section 2, we will summarize the property of the $L_{2}$-norm of the weak solutions to scalar conservation laws and define the functional for proving uniform boundedness of the weak solutions in $L_{1}$-norm for systems. In Section 3, we will discuss the generalized entropy functional both for invisid and viscous scalar conservation laws. The application of this functional in the invisid case to the stability of weak solutions to systems in $L_{1}$-norm will be briefly presented. And the relation of the generalized entropy functional between the invisid and viscous cases will be given for scalar conservation laws in the setting of shock and 
compression waves.

2. $L_{2}$-norm of the weak solution. For the general scalar conservation law (1.1), by choosing the particular convex entropy $\eta(u)=\frac{u^{2}}{2}$ with entropy flux $q(u)=$ $\int^{u} s f^{\prime}(s) d s$, we have the following entropy estimate, cf. [14].

LEMMA 2.1. Let $u(x, t)$ be a weak solution to the scalar conservation law (1.1) consisting of countable many admissible shocks $\left\{\alpha_{i}\right\}$, then

$$
\frac{d}{d t} \int u^{2}(x, t) d x=-2 \sum_{\alpha_{i}} A\left(\alpha_{i}\right),
$$

where the integral is over $\mathbf{R}$. Here, for an admissible shock $\alpha=\left(u^{-}, u^{+}\right), A(\alpha)$ denotes the area bounded by the curve $y=f(u)$ and the straight line segment connecting the end points $\left(u^{-}, f\left(u^{-}\right)\right)$and $\left(u^{+}, f\left(u^{+}\right)\right)$in the $u-y$ plane.

When the flux function is convex, the area $A(\alpha)$ of a shock wave $\alpha$ is of the order of $|\alpha|^{3}$ and the above estimate is the same as in $[8,9]$. The convexity of the flux function for a scalar conservation law corresponds to the genuine nonlinearity of the charactertistic field in a system, i.e. $r_{i} \cdot \nabla \lambda_{i} \neq 0$. Here $\lambda_{i}$ and $r_{i}$ are the eigenvalue and right eigenvector of the $i$-th family to a system of strictly hyperbolic conservation laws. The Hugoniot curves and rarefaction curves for systems are defined as follows.

For any state $u_{0}$, the Hugoniot curve $H\left(u_{0}\right)$ passing through $u_{0}$ is:

$$
H\left(u_{0}\right) \equiv\left\{u: \sigma\left(u_{0}, u\right)\left(u_{0}-u\right)=f\left(u_{0}\right)-f(u)\right\}
$$

for some scalar $\sigma\left(u_{0}, u\right)$. It follows easily from the strict hyperbolicity of the system that in a small neighborhood $\Omega$ of a state $u_{0}$, the set $H\left(u_{0}\right)$ consists of $n$ smooth curves $H_{i}\left(u_{0}\right), i=1,2, \cdots, n$, through $u_{0}$, satisfying that $\sigma\left(u_{0}, u\right)$ tends to $\lambda_{i}\left(u_{0}\right)$ as u moves along $H_{i}\left(u_{0}\right)$ toward $u_{0}$. A discontinuity $\left(u_{-}, u_{+}\right)$is called an $i$-discontinuity if $u_{+} \in H_{i}\left(u_{-}\right)$. (2.1) is called the Rankine-Hugoniot condition for discontinuties.

For general system, not necessarily genuinely nonlinear, the entropy condition to select the physically admissible weak solutions can be stated as follows, cf. [10].

Definition 1. A discontinuity $\left(u_{-}, u_{+}\right)$is admissible if

$$
\sigma_{i}\left(u_{-}, u_{+}\right) \leq \sigma_{i}\left(u_{-}, u\right)
$$

for any state $u$ on the Hugoniot curve $H\left(u_{-}\right)$between $u_{-}$and $u_{+}$.

The rarefaction curve of the $i$-th family passing through $u_{0}$ is the integral curve of $r_{i}(u)$ from this point, denoted by $R_{i}\left(u_{0}\right), i=1,2, \cdots, n$. The strict hyperbolicity also implies that $R_{i}\left(u_{0}\right), i=1,2, \cdots, n$, are $n$ smooth curves in a small neighborhood of $u_{0}$.

It is well-known that for the genuinely nonlinear characteristic field, the bifurcation of the Hugoniot curve from the rarefaction curve is of the third order of the shock wave strength. Thus, the decay estimate of the convex entropy gives the control of this kind of bifurcation.

When the flux function in (1.1) is noncovex, the authors in [14] show that the entropy estimate given in Lemma 2.1 is also closely related to the bifurcation of the Hugoniot curve from the rarefaction curve in the general system of hyperbolic conservation laws. In the following, we will explain this relation in details.

Consider the general system of strictly hyperbolic conservation laws in the form of (1.1) where both $u=\left(u^{1}, u^{2}, \cdots, u^{n}\right)$ and $f(u)$ are now $n$-vector functions. For 
illustration, we consider the wave of the first family and waves of the other families can be dealt with similarly. Up to a linear transformation, we assume that $u^{1}$ is a non-singular parameter along the 1-wave curve. For simplicity, we choose the right eigenvector corresponding to $\lambda_{1}$ as $r_{1}(u)=\left(1, \xi_{2}, \xi_{3}, \cdots, \xi_{n}\right)$.

For any state $u=\left(u^{1}, u^{2}, \cdots, u^{n}\right)$ along the rarefaction wave curve $R_{1}\left(u^{-}\right)$, we write

$$
u=u^{-}+\int_{u^{-, 1}}^{u^{1}} r_{1}(s) d s=\left(\begin{array}{c}
u^{1} \\
g\left(u^{1}\right)
\end{array}\right)
$$

for a smooth $(n-1)$-vector function $g\left(u^{1}\right)$. Similarly, for any state $u=$ $\left(u^{1}, u^{2}, \cdots, u^{n}\right) \in H_{1}\left(u^{-}\right)$, we write $u=\left(\begin{array}{c}u^{1} \\ h\left(u^{1}\right)\end{array}\right)$, for a smooth function $h\left(u^{1}\right)$.

Let $\alpha=\left(u^{-}, u^{+}\right)$be an admissible 1-shock to the system, and, without loss of generality, we assume that $u^{-, 1}<u^{+, 1}$. Then we have $s\left(u^{+}-u^{-}\right)=f\left(u^{+}\right)-f\left(u^{-}\right)$ for some scalar $s=\sigma\left(u^{-}, u^{+}\right)$. For any $u=\left(u^{1}, u^{2}, \cdots, u^{n}\right) \in H_{1}\left(u^{-}\right)$, we denote $s^{l}\left(u^{1}\right)=\sigma\left(u^{-}, u\right)$. Then by entropy condition for system, we have

$$
s^{l}\left(u^{1}\right) \geq s \quad \text { for } \quad u^{-, 1}<u^{1}<u^{+, 1} .
$$

If we consider the the scalar conservation law

$$
u_{t}^{1}+f_{x}^{1}\left(u^{1}, h\left(u^{1}\right)\right)=0
$$

then both the Rankine-Hugoniot condition and the Oleinik entropy condition [16] hold for the discontinuity $\left(u^{-, 1}, u^{+, 1}\right)$. That is, $\alpha^{1}=\left(u^{-, 1}, u^{+, 1}\right)$ is an admissible shock of (2.5). The following lemma shows that $\max _{u^{-, 1} \leq u^{1} \leq u^{+, 1}}\left|g\left(u^{1}\right)-h\left(u^{1}\right)\right|$ and $A\left(\alpha^{1}\right)$ defined for $\alpha^{1}$ as an admissible shock to the scalar conservation law (2.5) are of the same order.

LEMMA 2.2. Based on the above notations, we have

$$
\max _{u^{-, 1} \leq u^{1} \leq u^{+, 1}}\left|g\left(u^{1}\right)-h\left(u^{1}\right)\right|=0(1) A\left(\alpha^{1}\right) .
$$

One application of the estimate in Lemma 2.2 is to study the uniform boundedness of weak solutions in $L_{1}$-norm for general systems of hyperbolic conservation laws, [14]. To do this, we define a time-decreasing nonlinear functional $H[u(x, t)]$ as follows.

Given a solution $u(x, t)$ of the system (1.1), we define the pointwise distance along the rarefaction wave curves: solve the Riemann problem $(u(x, t), 0)$ by waves:

$$
u_{0}=u(x, t), u_{i} \in R_{i}\left(u_{i-1}\right), i=0,1, \cdots, n, u_{n}=0
$$

We set

$$
q_{i}(x, t) \equiv\left(u_{i}-u_{i-1}\right)^{i} .
$$

This way of assigning the distance is convenient in that $u^{i}$ is a conservative quantity and so it satisfies simple wave interaction estimates. For an $i$-wave $\alpha^{i}$ in the solution $u(x, t)$, we denote by $x\left(\alpha^{i}\right)=x\left(\alpha^{i}(t)\right)$ its location at time $t$. The linear part $L[u]$ of the nonlinear functional $H[u]$ is equivalent to the $L_{1}(x)$-norm of the solution:

$$
\begin{aligned}
& L[u(\cdot, t)] \equiv \sum_{i=1}^{n} L_{i}[u(\cdot, t)] \\
& L_{i}[u(\cdot, t)] \equiv \int_{-\infty}^{\infty}\left|q_{i}(x, t)\right| d x .
\end{aligned}
$$


We will use the notations $J(t)$ to denote the waves in the solution $u$ at a given time $t$. Moreover, $\alpha^{i}$ denotes a $i$-wave in $J(t)$. The other two components of the nonlinear functional $H[u]$, the quadratic $Q_{d}(t)$ and the convex entropy $E(t)$, are defined as follows:

$$
\begin{aligned}
& Q_{d}(t) \equiv Q_{d}[u(\cdot, t)]=\sum_{\alpha^{i} \in J(t)} Q_{d}\left(\alpha^{i}\right) \\
& Q_{d}\left(\alpha^{i}\right)=\left|\alpha^{i}\right|\left(\sum_{j>i} \int_{-\infty}^{x\left(\alpha^{i}\right)}\left|q_{j}(x, t)\right| d x+\sum_{j<i} \int_{x\left(\alpha^{i}\right)}^{\infty}\left|q_{j}(x, t)\right| d x\right) \\
& E(t) \equiv E[u(\cdot, t)]=\sum_{i=1}^{n} E_{i}(t)=\int_{-\infty}^{\infty}\left|q_{i}(x, t)\right|^{2} d x .
\end{aligned}
$$

Notice that $Q_{d}(t)$ defined above measures the coupling of waves propogating in different families. Combining the above nonlinear functionals, the nonlinear functional $H(t)$ for proving the uniform boundedness of weak solutions in $L_{1}$-norm can be defined as follows:

$$
H(t) \equiv H[u(\cdot, t)] \equiv\left(1+K_{1} F(t)\right) L(t)+K_{2}\left(Q_{d}(t)+E(t)\right),
$$

where $F(t)$ is the Glimm's functional measuring the wave interaction potential, cf. [5]. By appropriately choosing the constants $K_{1}$ and $K_{2}$, it is shown in [14] that the nonlinear functional $H(t)$ is non-increasing in time.

3. The generalized entropy. When we study the relation between two weak solutions, the $L_{2}$-norm of the difference of these two solutions does not give us any good estimates. In fact, any $L_{p}$-norm when $p>1$ is not approrpiate in this case. It can be seen by some very simple examples where compression waves become a shock wave that these norms increase in time even for scalar equations. In the study of existence and stability of weak solutions to general systems of hyperbolic conservation laws, the quantity

$$
|\alpha||\beta||\sigma(\alpha)-\sigma(\beta)|
$$

plays an important role. Here $\alpha$ and $\beta$ are two waves, and $\sigma(\alpha)$ and $\sigma(\beta)$ are their corresponding wave speeds. Notice that here $\alpha$ or $\beta$ may or may not be the wave in the two solutions under consideration. In the study of the global existence of weak solutions to a general system, the interaction potential of waves in the same family is defined [14] in the form of (3.1). And in the study of $L_{1}$ stability of weak solutions of systems whose characteristic fields are either genuinely nonlinear or linearly degenerate, a generalized entropy functional is introduced in [12] so that its time derivative gives an estimate of the quantities in the form of (3.1). In the following, we will give a description of this generalized entropy functional and study its property for viscous scalar conservation laws with convex flux.

We are interested in studying the nonlinear behavior of a genuinely nonlinear characteristic field in a system. For this, we consider a scalar conservation law (1.1) with $f^{\prime \prime}(u)>0$. In the following presentation, we assume for simplicity that the solutions are piecewise smooth. Thus we assume that two solutions $u_{1}(x, t)$ and $u_{2}(x, t)$ satisfy $u_{1}(x, t) \geq u_{2}(x, t)$ for $x_{2 i-1}<x<x_{2 i}(t)$, and $u_{1}(x, t) \leq u_{2}(x, t)$ for $x_{2 i}<x<x_{2 i+1}(t), i=0, \pm 1, \cdots$. For the $L_{1}$-norm of weak solutions to scalar conservation laws, the following theorem is well-known. 
THEOREM 3.1. Suppose that the difference of solutions $u_{1}(x, t)$ and $u_{2}(x, t)$ of the convex conservation law is a $L_{1}(x)$ function. Then there exists a positive constant $C$ depending only on $f(u)$ such that

$$
\frac{d}{d t} \int_{-\infty}^{\infty}\left|u_{1}(x, t)-u_{2}(x, t)\right| d x \leq-C \sum_{i} q_{i}^{u} q_{i}^{l}
$$

where $q_{i}^{u} q_{i}^{l}$ is zero if both solutions are continuous at $x_{i}(t)$ and is a second-order term if one of the solutions is discontinuous there;

$$
\begin{aligned}
& q_{i}^{u} \equiv\left\{\begin{array}{l}
\left(u_{1}\left(x_{i}(t)-0, t\right)-u_{2}\left(x_{i}(t), t\right)\right) \text { if } u_{1} \text { is discontinuous at } x=x_{i}(t), \\
\left(u_{2}\left(x_{i}(t)-0, t\right)-u_{1}\left(x_{i}(t), t\right)\right) \text { if } u_{2} \text { is discontinuous at } x=x_{i}(t)
\end{array}\right. \\
& q_{i}^{l} \equiv\left\{\begin{array}{l}
\left(u_{2}\left(x_{i}(t), t\right)-u_{1}\left(x_{i}(t)+0, t\right)\right) \text { if } u_{1} \text { is discontinuous at } x=x_{i}(t), \\
\left(u_{1}\left(x_{i}(t), t\right)-u_{2}\left(x_{i}(t)+0, t\right)\right) \text { if } u_{2} \text { is discontinuous at } x=x_{i}(t)
\end{array}\right.
\end{aligned}
$$

For a proof of this, see [13]. Note that the theorem implies the well-known $L_{1}(x)$ contraction semigroup property, which holds for scalar, not necessarily convex, conservation law.

On the other hand, it is easy to see that the solution operator is not a $L_{2}(x)$ contraction semigroup. To obtain the estimate for the terms in the form of (3.1) which is needed for the $L_{1}$ study, the authors in [12] introduce the following generalized entropy functional:

For any two functions $u$ and $v$, we set

$$
(u-v)^{+}=\left\{\begin{array}{ll}
u-v, & u \geq v, \\
0, & u<v,
\end{array} \quad(u-v)^{-}= \begin{cases}0, & u \geq v, \\
v-u, & u<v .\end{cases}\right.
$$

The generalized entropy functional $E(t)=E\left[u_{1}(\cdot, t), u_{2}(\cdot, t)\right]$ is defined as follows:

$$
\begin{aligned}
E(t)= & \int_{-\infty}^{\infty}\left|u_{1 y}\right|(y, t)\left(\int_{y}^{\infty}\left(u_{1}-u_{2}\right)^{+}(x, t) d x+\int_{-\infty}^{y}\left(u_{1}-u_{2}\right)^{-}(x, t) d x\right) d y \\
& +\int_{-\infty}^{\infty}\left|u_{2 y}\right|(y, t)\left(\int_{y}^{\infty}\left(u_{2}-u_{1}\right)^{+}(x, t) d x+\int_{-\infty}^{y}\left(u_{2}-u_{1}\right)^{-}(x, t) d x\right) d y
\end{aligned}
$$

The time derivative of the functional $E(t)$ can be stated as follows. The estimation of the above nonlinear functional in the BV framework is given in [4].

LEMMA 3.1. Let $u_{1}(x, t)$ and $u_{2}(x, t)$ be two solutions of $(1.1)$ with $f^{\prime \prime}(u)>0$ mentioned above. We have

$$
\begin{aligned}
\frac{d}{d t} E(t)= & -\int_{-\infty}^{\infty}\left|u_{1 y}(y, t)\right|\left(f\left(u_{2}(y, t)\right)-f\left(u_{1}(y, t)\right)\right. \\
& \left.+f^{\prime}\left(u_{1}(y, t)\right)\left(u_{1}(y, t)-u_{2}(y, t)\right)\right) d y \\
& -\int_{-\infty}^{\infty}\left|u_{2 y}(y, t)\right|\left(f\left(u_{1}(y, t)\right)-f\left(u_{2}(y, t)\right)\right. \\
& \left.+f^{\prime}\left(u_{2}(y, t)\right)\left(u_{2}(y, t)-u_{1}(y, t)\right)\right) d y,
\end{aligned}
$$

where $u_{i y}$ is a $\delta$-function at the location of a shock location. Let $u_{i}(x, t)$ have $a$ shock at $x_{0}$, then $\left|u_{i x}\left(x_{0}, t\right)\right|=\left(u_{i}\left(x_{0}-, t\right)-u_{i}\left(x_{0}+, t\right)\right) \delta\left(x-x_{0}\right)$, 
$f^{\prime}\left(u_{i}\right)\left(x_{0}, t\right)=\frac{f\left(u_{i}\left(x_{0}-\right)\right)-f\left(u_{i}\left(x_{0}+, t\right)\right)}{u_{i}\left(x_{0}-, t\right)-u_{i}\left(x_{0}+, t\right)}, f\left(u_{i}\right)\left(x_{0}, t\right)=\frac{f\left(u_{i}\left(x_{0}-\right)\right)+f\left(u_{i}\left(x_{0}+, t\right)\right)}{2}$ and $u_{i}\left(x_{0}, t\right)=\frac{u_{i}\left(x_{0}-\right)+u_{i}\left(x_{0}+, t\right)}{2}$.

A further analysis of the above estimate yields the following theorem for the decay of the generalized entropy functional.

THEOREM 3.2. Let $u_{i}(x, t), i=1,2$, be two solutions of $(1.1)$ with $f^{\prime \prime}(u)>0$ as stated in the beginning of this section. Then for any time $t$ when $u_{i}(x, t), i=1,2$, has no center rarefaction waves, we have

$$
\begin{aligned}
\frac{d}{d t} E(t) \leq C & \left\{\sum_{i=1}^{2} \int\left(u_{1}(x, t)-u_{2}(x, t)\right)^{2}\left|u_{i x}(x, t)\right| d x\right. \\
& \left.+\sum\left|\alpha_{i}(t)\right| \gamma_{x\left(\alpha_{i}(t)\right)}\left(\left|\alpha_{i}(t)\right|+\gamma_{x\left(\alpha_{i}(t)\right)}\right)\right\},
\end{aligned}
$$

where

$$
\gamma_{x(\beta(t))}=\left\{\begin{array}{rr}
\min \left\{\left|u_{1}(x(\beta(t)) \pm, t)-u_{2}(x(\beta(t)) \pm, t)\right|\right\} \\
x(\beta(t)) \neq x_{i}(t) & \text { for any } i \\
0, & x(\beta(t))=x_{i}(t)
\end{array}\right.
$$

Here the integral runs from the shock to shock in the two solutions, the summation is over all shock waves in both solutions, and $C$ is a positive constant.

Notice that the generalized entropy functional decreases except in the case when the two solutions behaves linearly to each other, i.e., when one solution contains only a shock and the other is the solution obtained by a shift in $x$-axis of the first solution. In this case, both solutions satisfy the following linear equation

$$
u_{t}+s u_{x}=0
$$

where $s$ is the shock speed. The estimation of the above functional uses mainly the convexity of the flux function. How to define a similar functional for a scalar conservation law with general flux function is not known and will be pursued by the authors.

When we study the $L_{1}$ distance between two weak solutions, it is more convenient to measure the pointwise distance by the Hugoniot curves. For this measurement, the following two lemmas are crucial and tell us that the terms in the form of (3.1) is the main error terms besides those corresponding to coupling of different families. Notice that the genuine nonlinearity of the characteristic field implies that

$$
|\sigma(\alpha)-\sigma(\beta)|=0(1)(|\alpha+\beta|),
$$

where $\alpha$ and $\beta$ are two waves in this family.

Lemma 3.2. Let $\bar{u} \in \Omega, \xi, \xi^{\prime} \in \mathbf{R}, k \in\{1, \cdots, n\}$. Define the states and the wave speeds

$$
\begin{aligned}
& u=H_{k}(\xi)(\bar{u}), \quad u^{\prime}=H_{k}\left(\xi^{\prime}\right)(u), \quad u^{\prime \prime}=H_{k}\left(\xi+\xi^{\prime}\right)(\bar{u}) \\
& \sigma=\sigma_{k}(\bar{u}, u), \quad \sigma^{\prime}=\sigma_{k}\left(u, u^{\prime}\right), \quad \sigma^{\prime \prime}=\sigma_{k}\left(\bar{u}, u^{\prime \prime}\right)
\end{aligned}
$$


Then we have

$$
\left|\left(\xi+\xi^{\prime}\right)\left(\sigma^{\prime \prime}-\sigma^{\prime}\right)-\xi\left(\sigma-\sigma^{\prime}\right)\right|=0(1) \cdot\left|\xi \xi^{\prime}\right|\left|\xi+\xi^{\prime}\right|
$$

LEMMA 3.3. If the values $\xi, \xi_{j}, \xi_{j}^{\prime}, j=1,2, \cdots, n$, satisfy

$$
\begin{aligned}
& H_{n}\left(\xi_{n}\right) \circ \cdots \circ H_{1}\left(\xi_{1}\right)(u) \\
& =\left\{\begin{array}{l}
H_{n}\left(\xi_{n}^{\prime}\right) \circ \cdots \circ H_{1}\left(\xi_{1}^{\prime}\right) \circ H_{i}(\xi)(u), \text { or } \\
H_{i}(\xi) \circ H_{n}\left(\xi_{n}^{\prime}\right) \circ \cdots \circ H_{1}\left(\xi_{1}^{\prime}\right)(u),
\end{array}\right.
\end{aligned}
$$

then

$$
\left|\xi_{i}-\xi_{i}^{\prime}-\xi\right|+\sum_{j \neq i}\left|\xi_{j}-\xi_{j}^{\prime}\right|=O(1)|\xi|\left(\left|\xi_{i}^{\prime}\right|\left|\xi_{i}^{\prime}+\xi\right|+\sum_{j \neq i}\left|\xi_{j}^{\prime}\right|\right)
$$

Based on the above observation, we can define the main nonlinear functional $H(t)$ for proving the $L_{1}$ stability of weak solutions to systems as follows:

$$
H(t) \equiv H[u(\cdot, t), v(\cdot, t)] \equiv\left(1+K_{1} F(t)\right) L(t)+K_{2}\left(Q_{d}(t)+E(t)\right),
$$

where $L(t), F(t)$ and $Q_{d}(t)$ have the same meaning as those stated in Section 2. By appropriately choosing the constants $K_{1}$ and $K_{2}$, the nonlinear functional $H(t)$ is shown to be non-increasing in time in [13].

Now we turn to study the viscous scalar conservation law.

$$
u_{t}+f(u)_{x}=\epsilon u_{x x}, \quad f^{\prime \prime}(u)>0,
$$

where $\epsilon$ is the constant viscosity coefficient.

Let $u_{i}(x, t)$ be two smooth solutions of (3.5) with $u_{1}(x, t)-u_{2}(x, t) \in L_{1}$ and $T V u_{i}(x, t)<\infty, i=1,2$. We can also define the generalized entropy functional $E^{\epsilon}(t)$ in the form of (3.3) for these two solutions. For the time derivative of $E^{\epsilon}(t)$, we have the following theorem.

TheOrem 3.3. Let $u_{1}(x, t)$ and $u_{2}(x, t)$ be two solutions of (3.5) mentioned above. We have

$$
\begin{aligned}
\frac{d}{d t} E^{\epsilon}(t) \leq & -\int_{-\infty}^{\infty}\left|u_{1 y}(y, t)\right|\left(f\left(u_{2}(y, t)\right)-f\left(u_{1}(y, t)\right)\right. \\
& \left.+f^{\prime}\left(u_{1}(y, t)\right)\left(u_{1}(y, t)-u_{2}(y, t)\right)\right) d y \\
& -\int_{-\infty}^{\infty}\left|u_{2 y}(y, t)\right|\left(f\left(u_{1}(y, t)\right)-f\left(u_{2}(y, t)\right)\right. \\
& \left.+f^{\prime}\left(u_{2}(y, t)\right)\left(u_{2}(y, t)-u_{1}(y, t)\right)\right) d y \\
& -2 \epsilon \int_{I_{1}^{+} \cap I_{2}^{+}}\left[\left(u_{1 y}\right)^{+}-\left(u_{2 y}\right)^{+}\right]^{2} d y+2 \epsilon \int_{I_{1}^{-} \cap I_{2}^{-}}\left[\left(u_{1 y}\right)^{-}-\left(u_{2 y}\right)^{-}\right]^{2} d y \\
& -2 \epsilon \int_{I_{1}^{+} \cap I_{2}^{-}}\left\{\left[\left(u_{1 y}\right)^{+}\right]^{2}-\left[\left(u_{2 y}\right)^{-}\right]^{2}\right\} d y \\
& +2 \epsilon \int_{I_{1}^{-} \cap I_{2}^{+}}\left\{\left[\left(u_{1 y}\right)^{-}\right]^{2}-\left[\left(u_{2 y}\right)^{+}\right]^{2}\right\} d y
\end{aligned}
$$


where

$$
I_{i}^{ \pm}=\left\{x \quad \mid \quad u_{i x} \geq(\leq) 0\right\}, \quad i=1,2 .
$$

Proof. For illustration, we give the estimation of $\frac{d}{d t} E^{\epsilon}(t)$ for one component of $E^{\epsilon}(t)$ in the interval $\left(x_{1}(t), x_{2}(t)\right)$. That is, we will estimate

$$
\frac{d}{d t} E_{11}^{\epsilon+}(t)=\int_{x_{1}(t)}^{x_{2}(t)}\left(u_{1}-u_{2}\right)(z, t)\left(\int_{-\infty}^{z}\left(u_{1 y}\right)^{+}(y, t) d y+\int_{z}^{\infty}\left(u_{2 y}\right)^{+}(y, t) d y\right) d z
$$

where we have used $u_{1}(x, t) \geq u_{2}(x, t)$ for $x_{1}(t)<x<x_{2}(t)$. For simplicity, we assume that $u_{1}(x, t)$ has a single local maximum at $x=\bar{y}_{1}(t)$ and $u_{2}(x, t)$ has a single local minimum at $x=\bar{y}_{2}(t)$ in $\left(x_{1}(t), x_{2}(t)\right)$. And let $x=y_{1}(t)$ be the first point on the left of $x_{1}(t)$ where $u_{1}(x, t)$ has a local minimum, and $x=y_{2}(t)$ be the first point on the right of $x_{2}(t)$ where $u_{2}(x, t)$ has a local maximum. Thus, $E_{11}^{\epsilon+}(t)$ can be rewritten as follows,

$$
\begin{aligned}
E_{11}^{\epsilon+}(t)= & \int_{x_{1}(t)}^{x_{2}(t)}\left(u_{1}-u_{2}\right)(z, t)\left(\int_{y_{1}(t)}^{z}\left(u_{1 y}\right)^{+}(y, t) d y+\int_{z}^{y_{2}(t)}\left(u_{2 y}\right)^{+}(y, t) d y\right) d z \\
& +\int_{x_{1}(t)}^{x_{2}(t)}\left(u_{1}-u_{2}\right)(z, t)\left(\int_{-\infty}^{y_{1}(t)}\left(u_{1 y}\right)^{+}(y, t) d y+\int_{y_{2}(t)}^{\infty}\left(u_{2 y}\right)^{+}(y, t) d y\right) d z .
\end{aligned}
$$

By noticing the signs of $u_{1 z z}$ and $u_{2 z z}$ at the points of local maximums and minimums, we know that

$$
\frac{d}{d t} \int_{-\infty}^{y_{1}(t)}\left(u_{1 y}\right)^{+}(y, t) d y \leq 0, \quad \frac{d}{d t} \int_{y_{2}(t)}^{\infty}\left(u_{2 y}\right)^{+}(y, t) d y \leq 0 .
$$

Furthermore, since

$$
\left(u_{1 z}-u_{2 z}\right)\left(x_{1}(t), t\right) \geq 0, \quad\left(u_{1 z}-u_{2 z}\right)\left(x_{2}(t), t\right) \leq 0
$$

we have

$$
\begin{aligned}
& \frac{d}{d t} \int_{x_{1}(t)}^{x_{2}(t)}\left(u_{1}-u_{2}\right)(z, t) d z \\
= & \left.\left(u_{1}-u_{2}\right)(z, t) \frac{d z}{d t}\right|_{z=x_{1}(t)} ^{z=x_{2}(t)}+\int_{x_{1}(t)}^{x_{2}(t)}\left(u_{1 t}(z, t)-u_{2 t}(z, t)\right) d z \\
= & \int_{x_{1}(t)}^{x_{2}(t)}\left\{-f\left(u_{1}(z, t)\right)_{z}+\epsilon u_{1 z z}(z, t)+f\left(u_{2}(z, t)\right)_{z}-\epsilon u_{2 z z}(z, t)\right\} d z \\
= & \epsilon\left(u_{1 z}-u_{2 z}\right)\left(x_{2}(t), t\right)-\epsilon\left(u_{1 z}-u_{2 z}\right)\left(x_{1}(t), t\right) \leq 0 .
\end{aligned}
$$

Combining (3.8) and (3.9) yields

$$
\frac{d}{d t}\left(\int_{-\infty}^{y_{1}(t)}\left(u_{1 y}\right)^{+}(y, t) d y+\int_{y_{2}(t)}^{\infty}\left(u_{2 y}\right)^{+}(y, t) d y\right) \int_{x_{1}(t)}^{x_{2}(t)}\left(u_{1}-u_{2}\right)(z, t) d z \leq 0 .
$$

Therefore, we have

$$
\begin{aligned}
\frac{d}{d t} E_{11}^{\epsilon+}(t) \leq & \frac{d}{d t} \int_{x_{1}(t)}^{x_{2}(t)}\left(u_{1}(z, t)-u_{2}(z, t)\right)\left(\int_{y_{1}(t)}^{z}\left(u_{1 y}\right)^{+}(y, t) d y\right. \\
& \left.+\int_{z}^{y_{2}(t)}\left(u_{2 y}\right)^{+}(y, t) d y\right) d z
\end{aligned}
$$


Now we will estimate (3.10) as follows. For convenience, we introduce the following notations.

$$
\phi_{1}^{+}(z, t)=\int_{y_{1}(t)}^{z}\left(u_{1 y}\right)^{+}(y, t) d y= \begin{cases}u_{1}(z, t)-u_{1}\left(y_{1}(t), t\right), & x_{1}(t) \leq z<\bar{y}_{1}(t) \\ u_{1}\left(\bar{y}_{1}(t), t\right)-u_{1}\left(y_{1}(t), t\right), & \bar{y}_{1}(t) \leq z \leq x_{2}(t)\end{cases}
$$

and

$$
\phi_{2}^{+}(z, t)=\int_{z}^{y_{2}(t)}\left(u_{2 y}\right)^{+}(y, t) d y= \begin{cases}u_{2}\left(y_{2}(t), t\right)-u_{2}\left(\bar{y}_{2}(t), t\right), & x_{1}(t) \leq z<\bar{y}_{2}(t) \\ u_{2}\left(y_{2}(t), t\right)-u_{2}(z, t), & \bar{y}_{2}(t) \leq z \leq x_{2}(t)\end{cases}
$$

Then we have

$$
\begin{aligned}
\frac{d}{d t} E_{11}^{\epsilon+}(t) \leq & \frac{d}{d t} \int_{x_{1}(t)}^{x_{2}(t)}\left(u_{1}-u_{2}\right)\left(\phi_{1}^{+}+\phi_{2}^{+}\right)(z, t) d z \\
= & \int_{x_{1}(t)}^{x_{2}(t)}\left\{\left(u_{1 t}-u_{2 t}\right)\left(\phi_{1}^{+}+\phi_{2}^{+}\right)+\left(u_{1}-u_{2}\right)\left(\phi_{1 t}^{+}+\phi_{2 t}^{+}\right)\right\}(z, t) d z \\
= & \int_{x_{1}(t)}^{x_{2}(t)}\left(-f\left(u_{1}\right)_{z}+\epsilon u_{1 z z}+f\left(u_{2}\right)_{z}-\epsilon u_{2 z z}\right)\left(\phi_{1}^{+}+\phi_{2}^{+}\right)(z, t) d z \\
& +\int_{x_{1}(t)}^{\bar{y}_{1}(t)}\left(u_{1}-u_{2}\right)(z, t)\left(u_{1 t}(z, t)-\frac{d}{d t} u_{1}\left(y_{1}(t), t\right)\right) d z \\
& +\int_{\bar{y}_{1}(t)}^{x_{2}(t)}\left(u_{1}-u_{2}\right)(z, t)\left(\frac{d}{d t} u_{1}\left(\bar{y}_{1}(t), t\right)-\frac{d}{d t} u_{1}\left(y_{1}(t), t\right)\right) d z \\
& +\int_{x_{1}(t)}^{\bar{y}_{2}(t)}\left(u_{1}-u_{2}\right)(z, t)\left(\frac{d}{d t} u_{2}\left(\bar{y}_{2}(t), t\right)-\frac{d}{d t} u_{2}\left(y_{2}(t), t\right)\right) d z \\
& +\int_{\bar{y}_{2}(t)}^{x_{2}(t)}\left(u_{1}-u_{2}\right)(z, t)\left(\frac{d}{d t} u_{2}\left(\bar{y}_{2}(t), t\right)-u_{2 t}(z, t)\right) d z
\end{aligned}
$$

Notice that

$$
\begin{aligned}
\frac{d}{d t} u_{1}\left(y_{1}(t), t\right) & \geq 0, & \frac{d}{d t} u_{1}\left(\bar{y}_{1}(t), t\right) & \leq 0 \\
\frac{d}{d t} u_{2}\left(y_{2}(t), t\right) & \leq 0 & \frac{d}{d t} u_{2}\left(\bar{y}_{2}(t), t\right) & \geq 0
\end{aligned}
$$

and

$$
\phi_{1 z}^{+}(z, t)= \begin{cases}u_{1 z}(z, t), & x_{1}(t) \leq z<\bar{y}_{1}(t) \\ 0, & \bar{y}_{1}(t) \leq z \leq x_{2}(t)\end{cases}
$$

and

$$
\phi_{2 z}^{+}(z, t)= \begin{cases}0, & x_{1}(t) \leq z<\bar{y}_{2}(t) \\ -u_{2 z}(z, t), & \bar{y}_{2}(t) \leq z \leq x_{2}(t)\end{cases}
$$


By some calculations, we have

$$
\begin{aligned}
\frac{d}{d t} E_{11}^{\epsilon+}(t) \leq & \int_{x_{1}(t)}^{\bar{y}_{1}(t)}\left(f\left(u_{1}\right)-f\left(u_{2}\right)\right) u_{1 z}(z, t) d z-\int_{\bar{y}_{2}(t)}^{x_{2}(t)}\left(f\left(u_{1}\right)-f\left(u_{2}\right)\right) u_{2 z}(z, t) d z \\
& -2 \epsilon \int_{x_{1}(t)}^{\bar{y}_{1}(t)}\left(u_{1 z}-u_{2 z}\right) u_{1 z}(z, t) d z+2 \epsilon \int_{\bar{y}_{2}(t)}^{x_{2}(t)}\left(u_{1 z}-u_{2 z}\right) u_{2 z}(z, t) d z \\
& -\int_{x_{1}(t)}^{\bar{y}_{1}(t)}\left(u_{1}-u_{2}\right) f^{\prime}\left(u_{1}\right) u_{1 z}(z, t) d z+\int_{\bar{y}_{2}(t)}^{x_{2}(t)}\left(u_{1}-u_{2}\right) f^{\prime}\left(u_{2}\right) u_{2 z}(z, t) d z \\
= & -\int_{x_{1}(t)}^{x_{2}(t)}\left\{\left(u_{1 z}\right)^{+}\left[f\left(u_{2}\right)-f\left(u_{1}\right)-f^{\prime}\left(u_{1}\right)\left(u_{2}-u_{1}\right)\right]\right. \\
& \left.+\left(u_{2 z}\right)^{+}\left[f\left(u_{1}\right)-f\left(u_{2}\right)-f^{\prime}\left(u_{2}\right)\left(u_{1}-u_{2}\right)\right]\right\} d z \\
& -2 \epsilon \int_{I_{1,1}^{+} \cap I_{2,1}^{+}}\left[\left(u_{1 z}\right)^{+}-\left(u_{2 z}\right)^{+}\right]^{2} d z-2 \epsilon \int_{I_{1,1}^{+} \cap I_{2,1}^{-}}\left(u_{1 z}\right)^{+}\left[\left(u_{1 z}\right)^{+}+\left(u_{2 z}\right)^{-}\right] d z \\
& -2 \epsilon \int_{I_{1,1}^{-} \cap I_{2,1}^{+}}\left(u_{2 z}\right)^{+}\left[\left(u_{1 z}\right)^{-}-\left(u_{2 z}\right)^{+}\right] d z
\end{aligned}
$$

where

$$
I_{i, 1}^{ \pm}=I_{i}^{ \pm} \cap\left[x_{1}(t), x_{2}(t)\right], \quad i=1,2
$$

Similar estimation applies to

$$
E_{11}^{\epsilon-}=\int_{x_{1}(t)}^{x_{2}(t)}\left(u_{1}-u_{2}\right)(z, t)\left(\int_{-\infty}^{z}\left(u_{1 y}\right)^{-}(y, t) d y+\int_{z}^{\infty}\left(u_{2 y}\right)^{-}(y, t) d y\right) d z
$$

and other components in the nonlinear functional $E^{\epsilon}(t)$. Combining all these estimates completes the proof of Theorem 3.3.

Now we are ready to compare $\frac{d}{d t} E(t)$ and $\frac{d}{d t} E^{\epsilon}(t)$. Since the generalized nonlinear functional captures mainly the nonlinearity of the scalar conservation laws and reveals the nonlinear evolution of the weak solutions, it decreases more rapidly for compression waves than a shock of the same strength. When $\epsilon>0$, all the shocks for $\epsilon=0$ are smoothed out to be compression waves. Hence, $\frac{d}{d t} E^{\epsilon}(t)$ has more terms than $\frac{d}{d t} E(t)$ and some of the extra terms do not approach to zero as $\epsilon$ tends to zero.

Denote the right hand side of (3.6) for $\frac{d}{d t} E^{\epsilon}(t)$ by $D_{1}^{\epsilon}+D_{2}^{\epsilon}$, where

$$
\begin{aligned}
D_{1}^{\epsilon}=- & \int_{-\infty}^{\infty}\left|u_{1 y}(y, t)\right|\left(f\left(u_{2}(y, t)\right)-f\left(u_{1}(y, t)\right)\right. \\
& \left.+f^{\prime}\left(u_{1}(y, t)\right)\left(u_{1}(y, t)-u_{2}(y, t)\right)\right) d y \\
- & \int_{-\infty}^{\infty}\left|u_{2 y}(y, t)\right|\left(f\left(u_{1}(y, t)\right)-f\left(u_{2}(y, t)\right)\right. \\
& \left.+f^{\prime}\left(u_{2}(y, t)\right)\left(u_{2}(y, t)-u_{1}(y, t)\right)\right) d y
\end{aligned}
$$


and

$$
\begin{aligned}
D_{2}^{\epsilon}= & -2 \epsilon \int_{I_{1}^{+} \cap I_{2}^{+}}\left[\left(u_{1 y}\right)^{+}-\left(u_{2 y}\right)^{+}\right]^{2} d y+2 \epsilon \int_{I_{1}^{-} \cap I_{2}^{-}}\left[\left(u_{1 y}\right)^{-}-\left(u_{2 y}\right)^{-}\right]^{2} d y \\
& -2 \epsilon \int_{I_{1}^{+} \cap I_{2}^{-}}\left\{\left[\left(u_{1 y}\right)^{+}\right]^{2}-\left[\left(u_{2 y}\right)^{-}\right]^{2}\right\} d y \\
& +2 \epsilon \int_{I_{1}^{-} \cap I_{2}^{+}}\left\{\left[\left(u_{1 y}\right)^{-}\right]^{2}-\left[\left(u_{2 y}\right)^{+}\right]^{2}\right\} d y
\end{aligned}
$$

Notice that $D_{1}^{\epsilon}$ is in the same form as the right hand side of (3.4) for $\frac{d}{d t} E(t)$ which is denoted by $D_{1}$. We will show the following equality

$$
\lim _{\epsilon \rightarrow 0}\left(D_{1}^{\epsilon}+D_{2}^{\epsilon}\right)=D_{1}
$$

holds in a simple illustrative setting. This implies the extra terms in $\frac{d}{d t} E^{\epsilon}(t)$ compensate the difference of the decay rates of $\frac{d}{d t} E(t)$ for compression waves and the corresponding shocks. The rigorous proof of (3.13) for the general case is beyond the scope of this paper and left for the future study.

Let $u_{1}(x, t)$ be a travelling wave solution to (3.5) with speed $s$ and the end states at $x= \pm \infty$ be $u_{ \pm}$respectively. The corresponding solution for (1.1) when $\epsilon=0$ is a single shock connecting states $\left(u_{-}, u_{+}\right)$with speed $s$. Let the solution $u_{2}(x, t)$ be a smooth solution to (3.5) which converges to the solution $u_{2}(x, t)$ when $\epsilon=0$ with uniformly bounded derivatives in $x$ with respect to $\epsilon$ everywhere. Without any ambiguity, we denote both the solutions for $\epsilon>0$ and $\epsilon=0$ by $u_{i}(x, t), i=1,2$.

Under the above assumption, we can estimate $D_{2}^{\epsilon}$ as follows. Let $u_{1}(x, t)=$ $\eta\left(\frac{x-s t}{\epsilon}\right)$ and plug this into the equation (3.5). By integration once, we have

$$
\left(\eta^{\prime}\right)^{2}=\left[f(\eta)-s \eta-f\left(u_{-}\right)+s u_{-}\right] \eta^{\prime}
$$

Thus,

$$
\begin{aligned}
\lim _{\epsilon \rightarrow 0} D_{2}^{\epsilon} & =2 \lim _{\epsilon \rightarrow 0} \epsilon \int_{-\infty}^{\infty}\left[\left(u_{1 z}\right)^{-}\right]^{2} d z \\
& =2 \lim _{\epsilon \rightarrow 0} \epsilon^{-1} \int_{-\infty}^{\infty}\left[f(\eta)-s \eta-f\left(u_{-}\right)+s u_{-}\right] \eta^{\prime} d z \\
& =-2 \int_{-\infty}^{\infty}\left|u_{1 z}\right|\left[f\left(u_{1}\right)-\frac{f\left(u_{-}\right)-f\left(u_{+}\right)}{u_{-}-u_{+}}\left(u_{1}-u_{-}\right)-f\left(u_{-}\right)\right] d z \\
& =-2 \int_{u_{+}}^{u_{-}} f(s) d s+\left(u_{-}-u_{+}\right)\left[f\left(u_{-}\right)+f\left(u_{+}\right)\right]
\end{aligned}
$$

Now we turn to estimate $D_{1}^{\epsilon}-D_{1}$. Notice that the only difference comes from the 
compression waves becoming a shock in $u_{1}(x, t)$. We have

$$
\begin{aligned}
& \lim _{\epsilon \rightarrow 0} D_{1}^{\epsilon}-D_{1} \\
= & -\int_{-\infty}^{\infty}\left|u_{1 z}\right|\left(f\left(u_{2}\right)-f\left(u_{1}\right)-f^{\prime}\left(u_{1}\right)\left(u_{2}-u_{1}\right)\right)(z, t) d z \\
& +\int_{-\infty}^{\infty}\left|u_{1 z}\right|\left(f\left(u_{2}\right)-\frac{f\left(u_{+}\right)+f\left(u_{-}\right)}{2}-\frac{f\left(u_{+}\right)-f\left(u_{-}\right)}{u_{+}-u_{-}}\left(u_{2}-\frac{u_{+}+u_{-}}{2}\right)\right)(z, t) d z \\
= & 2 \int_{u_{+}}^{u_{-}} f(s) d s-\left(u_{-}-u_{+}\right)\left[f\left(u_{-}\right)+f\left(u_{+}\right)\right] .
\end{aligned}
$$

Combining (3.14) and (3.15) yields (3.13) for this case.

By constructing a nonlinear Lyapunov functional which controls the area swept by a curve moving in a plane in the direction of curvature, the authors in [2] give a priori estimates on solutions to a class of parabolic equations and of scalar conservation laws. For a special class of systems of strictly hyperboic system with small viscosity, the authors in [1] study the well-posedness of the vanishing viscosity solutions. Whether the nonlinear functional $E^{\epsilon}(t)$ defined above can be used for the study of systems of viscous conservation laws is not known and left for future investigation.

Acknowledgement: The research of the first author was supported in part by NSF Grant DMS-9803323. The research of the second author was supported in part by the RGC Competitive Earmarked Research Grant 9040468(CityU 1087/99P). And the authors would like to thank Professor Zhouping Xin for the interesting discussion on the generalized entropy functional for the viscous scalar conservation laws.

\section{REFERENCES}

[1] BiAnChInI, S. AND BREsSAn, A., Vanishing viscosity solutions of hyperbolic systems on manifolds, preprint.

[2] BiAnchini, S. AND BRESSAN, A., On a Lyapunov functional relating shortening curves and viscous conservation laws, preprint.

[3] Bressan, A., Goatin, G., And PiCcoli B., Well posedness of the Cauchy problem for $n \times n$ systems of conservation laws, to appear in Memoir, Amer. Math. Soc.

[4] Dafermos, C., "Hyperbolic Conservation Laws in Continuum Physics", Springer-Verlag 2000.

[5] Glimm, J., Solutions in the large for nonlinear hyperbolic systems of equations, Comm. Pure Appl. Math., 18 (1965), pp. 697-715.

[6] KRuzKov, S., First-order quasilinear equations with several space variables, Mat. Sb., 123 (1970), pp. 228-255; English transl. in Math. USSR Sb., 10 (1970), pp. 217-273.

[7] LAx, P.D., Hyperbolic systems of conservation laws II, Comm. Pure Appl. Math., 10 (1957), pp. 537-566.

[8] LAx, P.D., Shock waves and entropy, Contribution to Nonlinear Functional Analysis, (E. Zarantonello,ed.), Academic Press, N.Y. 1971, pp. 603-634.

[9] LAx, P.D., Hyperbolic systems of conservation laws and the mathematical theory of shock waves, CBMS. 11, SIAM, 1973.

[10] LiU, T.-P., The entropy condition and the admissibility of shocks, J. Math. Anal. Appl., 53 (1976), pp. 78-88.

[11] LiU, T.-P. AND YANG, T., Uniform $L_{1}$ boundedness of solutions of hyperbolic conservation laws, Methods and Appl. Anal., 67 (1997), no.3-4, pp. 313-326.

[12] LiU, T.-P. AND YANG, T., A new entropy functional for scalar conservation laws, Comm. Pure Appl. Math., 52 (1999), no.11, pp. 1427-1442.

[13] LiU, T.-P. AND YANG, T., Well-posedness theory for hyperbolic conservation laws, Comm. Pure Appl. Math., 52 (1999), no. 12, pp. 1553-1586.

[14] LIU, T.-P. AND YANG, T., Weak solutions of general systems of hyperbolic conservation laws, preprint. 
[15] Keyfitz, B., Solutions with shocks: An example of $L_{1}$-contractive semigroup, Comm. Pure Appl. Math., 24 (1971), pp. 125-132.

[16] OLEINIK, O., Uniqueness and stability of the generalized solution of the Cauchy problem for a quasilinear equation, Usp. Mat. Nauk, 14 (1959), pp. 165-170; Amer. Math. Soc. Transl., Ser. 2, 33 (1964), pp. 285-290.

[17] Schaeffer, D., A regularity theorem for conservation laws, Adv. Math., 11 (1973), pp. 368386.

[18] Smoller, J., Shock waves and reaction-diffusion equations, Springer-Verlag, New York, 1982.

[19] Temple, B., Systems of conservation laws with invariant submanifolds, Trans. Amer. Math. Soc., 280 (1983), pp. 781-795.

[20] TARTAR, L., Compensated compactness and applications to partial differential equations, Research Notes in mathematics, Nonlinear Analysis and Mechanics, (R.J. Knops, ed.) Vol. 4, Pitman Press, New York, 1979. 